Sādhanā Vol. 39, Part 3, June 2014, pp. 573-581. (C) Indian Academy of Sciences

\title{
A method for unbalanced transportation problems in fuzzy environment
}

\author{
DEEPIKA RANI ${ }^{1, *}$, T R GULATI $^{1}$ and AMIT KUMAR ${ }^{2}$ \\ ${ }^{1}$ Department of Mathematics, Indian Institute of Technology Roorkee, \\ Roorkee 247 667, India \\ ${ }^{2}$ School of Mathematics and Computer Applications, Thapar University, \\ Patiala 147 004, India \\ e-mail: deepikaiitroorkee@gmail.com; trgulati@gmail.com; amitkdma@gmail.com
}

MS received 6 September 2013; revised 7 January 2014; accepted 25 January 2014

\begin{abstract}
In this paper, we consider the fully fuzzy unbalanced transportation problem in which the total availability/production is more than the total demand and propose a method to solve it. Such problems are usually solved by adding a dummy destination. Since the dummy destination has no existence in reality, the excess availability is not transported at all and is held back at one or more origins. The method proposed in this paper gives the additional information that to which of the destination(s) the excess availability be transported for future demand at minimum cost. The advantage of the proposed method over the existing method is that the fuzzy optimal solution obtained does not involve the dummy destination. The method has been illustrated with the help of an example.
\end{abstract}

Keywords. Trapezoidal fuzzy number; fully fuzzy transportation problem; fuzzy optimal solution.

\section{Introduction}

Among linear programming problems, the transportation problem is very popular. As transportation of goods from one place to another becomes increasingly important in global economics, knowledge of the transportation system is fundamental to the efficient and economical operation of a company. Transportation models play an important role in reducing cost and improving service. It ensures the efficient movement of raw materials and finished goods. It is one of the earliest applications of linear programming problems.

Hitchcock (1941) originally developed the basic transportation problem. Appa (1973) discussed several variations of the transportation problem. In general, transportation problems are solved with the assumptions that the parameters of the transportation problem (i.e., unit cost of transportation from each source to each destination, availability of the product at each source and demand at each destination) are specified in a precise way, i.e., in crisp environment. But in

*For correspondence 
practice, the parameters of the transportation problem are not always exactly known and stable. This imprecision may follow from the lack of exact information, uncertainty in judgment etc. Therefore, Zadeh (1965) introduced the concept of fuzzy numbers.

Saad \& Abbas (2003) discussed an algorithm for solving the transportation problems in fuzzy environment. Das \& Baruah (2007) proposed vogel's approximation method to find the fuzzy initial basic feasible solution of fuzzy transportation problems in which all the parameters are represented by triangular fuzzy numbers. Basirzadeh (2011) used the classical algorithms to find the fuzzy optimal solution of fully fuzzy transportation problems by transforming the fuzzy parameters into crisp parameters.

Pandian \& Natrajan (2010) proposed a new algorithm, namely fuzzy zero point method, to find the fuzzy optimal solution of fuzzy transportation problems, where trapezoidal fuzzy numbers represent all the parameters. Later, based on fuzzy zero point method, they proposed a new method to find more-for-less fuzzy optimal solution for such fuzzy transportation problems which have mixed constraints and parameters as trapezoidal fuzzy numbers (Pandian \& Natrajan 2010a). De \& Yadav (2010) modified the existing method (Kikuchi 2000) by using trapezoidal fuzzy numbers instead of triangular fuzzy numbers. Kaur \& Kumar (2011) proposed a new method for the fuzzy transportation problems using ranking function. Using simplex- type algorithm proposed by Arsham \& Khan (1989), Gani et al (2011) obtained the fuzzy optimal solution of fuzzy transportation problems having parameters as trapezoidal fuzzy numbers.

While solving unbalanced transportation problems we come across two type of cases. Either the total availability is more than the total demand or vice-versa. In case of excess availability, some times it may happen that we do not have enough storage place for the excess commodity at the source(s) and wish to transport it to the destination(s) for the future demand. To solve such problems a dummy destination is added, where the excess availability is transported. Since the dummy destination does not have any existence in reality, so it is not possible to find that the excess available product should be transported to which destination at a minimum cost. In this paper, a method is proposed to get the fuzzy optimal solution in terms of original sources and destinations only and we also get the above said information. To illustrate the proposed method, a fully fuzzy transportation problem is solved using the proposed method and the obtained results are discussed.

\section{Prelminaries}

In this section, some basic definitions, arithmetic operations and comparison of trapezoidal fuzzy numbers are presented.

\subsection{Basic definitions}

Definition 1 (Kaufmann \& Gupta 1985). A fuzzy number $\widetilde{A}$ defined on the universal set of real numbers $\mathbb{R}$, denoted as $\widetilde{A}=(a, b, c, d)$, is said to be a trapezoidal fuzzy number if its membership function $\mu_{\widetilde{A}}(x)$ is given by

$$
\mu_{\widetilde{A}}(x)=\left\{\begin{array}{cl}
\frac{(x-a)}{(b-a)}, & a \leq x<b \\
1, & b \leq x \leq c \\
\frac{(d-x)}{(d-c)}, & c<x \leq d \\
0, & \text { otherwise. }
\end{array}\right.
$$

Graphically, a trapezoidal fuzzy number can be represented as (figure 1): 


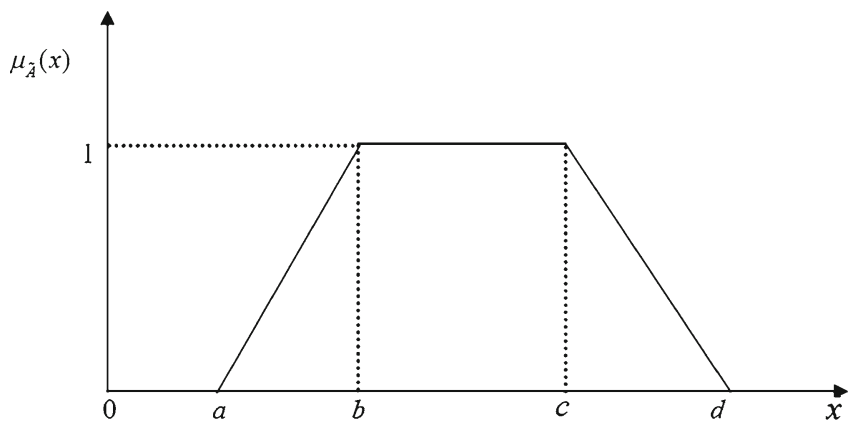

Figure 1. Trapezoidal fuzzy number.

Definition 2 (Liou \& Wang 1992). Let $F(\mathbb{R})$ be a set of fuzzy numbers defined on the set of real numbers $\mathbb{R}$ and let $\widetilde{A}=(a, b, c, d) \in F(\mathbb{R})$. Then

$$
\Re(\widetilde{A})=\frac{(a+b+c+d)}{4},
$$

is called a ranking function, which maps each fuzzy number into real line.

Remark 1. A trapezoidal fuzzy number $\widetilde{A}=(a, \underset{\sim}{,}, c, d)$ is said to be zero trapezoidal fuzzy number if and only if $\Re(\widetilde{A})=0$ and is denoted by $\widetilde{0}$.

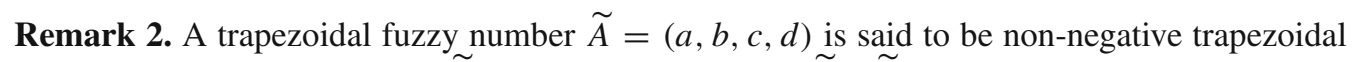
fuzzy number if and only if $\Re(\widetilde{A}) \geq 0$ and is denoted by $\widetilde{A} \succeq \widetilde{0}$.

\subsection{Arithmetic operations (Kaufmann \& Gupta 1985)}

Let $\widetilde{A}_{1}=\left(a_{1}, b_{1}, c_{1}, d_{1}\right)$ and $\widetilde{A}_{2}=\left(a_{2}, b_{2}, c_{2}, d_{2}\right)$ be two trapezoidal fuzzy numbers. Then

(i) $\widetilde{A}_{1} \oplus \widetilde{A}_{2}=\left(a_{1}+a_{2}, b_{1}+b_{2}, c_{1}+c_{2}, d_{1}+d_{2}\right)$,

(ii) $\widetilde{A}_{1} \ominus \widetilde{A}_{2}=\left(a_{1}-d_{2}, b_{1}-c_{2}, c_{1}-b_{2}, d_{1}-a_{2}\right)$,

(iii) $k \tilde{A}_{1}= \begin{cases}\left(k a_{1}, k b_{1}, k c_{1}, k d_{1}\right), & k \geq 0 \\ \left(k d_{1}, k c_{1}, k b_{1}, k a_{1}\right), & k \leq 0,\end{cases}$

(iv) $\widetilde{A}_{1} \otimes \widetilde{A}_{2}=(a, b, c, d)$,

where $a=\min \left(a_{1} a_{2}, a_{1} d_{2}, d_{1} a_{2}, d_{1} d_{2}\right), b=\min \left(b_{1} b_{2}, b_{1} c_{2}, c_{1} b_{2}, c_{1} c_{2}\right), c=\max \left(b_{1} b_{2}\right.$, $\left.b_{1} c_{2}, c_{1} b_{2}, c_{1} c_{2}\right), d=\max \left(a_{1} a_{2}, a_{1} d_{2}, d_{1} a_{2}, d_{1} d_{2}\right)$.

\subsection{Comparison of trapezoidal fuzzy numbers (Liou \& Wang 1992)}

Let $F(\mathbb{R})$ be a set of fuzzy numbers. Let $\widetilde{A}_{1}=\left(a_{1}, b_{1}, c_{1}, d_{1}\right)$ and $\widetilde{A}_{2}=\left(a_{2}, b_{2}, c_{2}, d_{2}\right)$ be two trapezoidal fuzzy numbers. Then

$$
\begin{aligned}
& \widetilde{A}_{1} \succ \widetilde{A}_{2} \text { if } \Re\left(\widetilde{A}_{1}\right)>\Re\left(\widetilde{A}_{2}\right) \\
& \widetilde{A}_{1} \prec \widetilde{A}_{2} \text { if } \Re\left(\widetilde{A}_{1}\right)<\Re\left(\widetilde{A}_{2}\right) \\
& \widetilde{A}_{1} \approx \widetilde{A}_{2} \text { if } \Re\left(\widetilde{A}_{1}\right)=\Re\left(\widetilde{A}_{2}\right) .
\end{aligned}
$$




\section{Fuzzy linear programming formulation}

Consider an unbalanced FFTP having $m$ sources $S_{i}$ with fuzzy availability $\widetilde{a}_{i}(1 \leq i \leq m), n$ destinations $D_{j}$ with fuzzy demand $\widetilde{b}_{j}(1 \leq j \leq n)$. Each of the $m$ sources $S_{i}$ can transport to any of the $n$ destinations $D_{j}$ at a transportation cost of $\widetilde{c}_{i j}$ per unit. Let $\widetilde{x}_{i j}$ be the fuzzy quantity of the product that should be transported from source $S_{i}$ to destination $D_{j}$. The linear programming formulation to minimize the total fuzzy transportation cost is as follows:

$$
\begin{aligned}
(L P 1) \quad \text { Minimize } \quad & \sum_{i=1}^{m} \sum_{j=1}^{n}\left(\widetilde{c}_{i j} \otimes \tilde{x}_{i j}\right), \quad \text { subject to } \\
& \sum_{j=1}^{n} \tilde{x}_{i j} \succeq \widetilde{a}_{i} \quad(1 \leq i \leq m), \\
& \sum_{i=1}^{m} \tilde{x}_{i j} \succeq \widetilde{b}_{j} \quad(1 \leq j \leq n), \\
& \tilde{x}_{i j} \succeq \tilde{0} .
\end{aligned}
$$

\section{Solution by existing methods}

Example 1. Consider the transportation problem with 3 sources $S_{i}(1 \leq i \leq 3)$ and 3 destinations $D_{j}(1 \leq j \leq 3)$ with unit costs of transporting the product from source $S_{i}$ to destination $D_{j}$ as

$$
\left[\widetilde{c}_{i j}\right]=\left[\begin{array}{ccc}
(4,5,8,15) & (1,1,2,4) & (1,2,3,6) \\
(1,1,2,4) & (7,9,12,20) & (4,5,8,15) \\
(0,1,1,2) & (7,12,15,26) & (1,1,2,4)
\end{array}\right]
$$

The fuzzy availability of the product at first, second and third sources is $(9,11,20,40),(3,4,7,14)$ and $(3,4,5,8)$, respectively and $(7,12,15,26),(4,8,10,18)$ and $(2,5,6,11)$ is the fuzzy demand of the product at first, second and third destinations, respectively. The product can be shipped from any source to any destination. One has to find the fuzzy quantity of the product that should be transported from each source to each destination such that the total fuzzy cost of transportation is minimum.

The chosen problem can be represented in tabular form as shown in table 1 .

Table 1. Unbalanced fully fuzzy transportation problem.

\begin{tabular}{lcccc}
\hline $\begin{array}{l}\text { Destinations } \rightarrow \\
\text { Sources } \downarrow\end{array}$ & $D_{1}$ & $D_{2}$ & $D_{3}$ & Fuzzy availability \\
\hline$S_{1}$ & $(4,5,8,15)$ & $(1,1,2,4)$ & $(1,2,3,6)$ & $(9,11,20,40)$ \\
$S_{2}$ & $(1,1,2,4)$ & $(7,9,12,20)$ & $(4,5,8,15)$ & $(3,4,7,14)$ \\
$S_{3}$ & $(0,1,1,2)$ & $(7,12,15,26)$ & $(1,1,2,4)$ & $(3,4,5,8)$ \\
Fuzzy demand & $(7,12,15,26)$ & $(4,8,10,18)$ & $(2,5,6,11)$ & \\
\hline
\end{tabular}


For the above transportation problem the total fuzzy availability is more than the total fuzzy demand. So we first convert it to a balanced problem by adding a dummy destination $\left(D_{4}\right)$ with the corresponding unit transportation costs to be zero trapezoidal fuzzy numbers and convert the constraint inequalities to equations. Now on solving this balanced problem by the existing methods (Basirzadeh 2011; Pandian \& Natrajan 2010, 2010a), the following fuzzy optimal solution is obtained:

$\tilde{x}_{11}=(-15,0,7,20), \tilde{x}_{12}=(4,8,10,18), \tilde{x}_{14}=(-40,-12,7,49), \tilde{x}_{21}=(3,4,7,14), \tilde{x}_{31}=(3$,

$4,5,8)$ and the remaining $\tilde{x}_{i j}$ to be zero trapezoidal fuzzy numbers. Total fuzzy transportation cost is found to be $(-216,26,113,510)$.

From the above results, we find that the fuzzy quantity $(-40,-12,7,49)$ is to be transported to dummy destination $D_{4}$ from source $S_{1}$ to balance the excess availability. Since, dummy destination has no significance in reality, so the existing methods (Basirzadeh 2011; Pandian \& Natrajan 2010, 2010a) do not give any further information.

\section{Proposed method}

In this section, a method is proposed to solve the unbalanced FFTPs in which total availability is more than total demand. We first prove the following result.

Theorem 1. Let ( $L P 2)$ be the fuzzy linear formulation of balanced FFTP obtained by adding a dummy source $S_{m+1}$ with unit fuzzy transportation costs $\widetilde{c}_{(m+1) j}=\min _{1 \leq i \leq m}\left(\widetilde{c}_{i j}\right), 1 \leq j \leq$ $n$, a dummy destination $D_{n+1}$ with $\widetilde{c}_{i(n+1)}=\min _{1 \leq j \leq n}\left(\widetilde{c}_{i j}\right), 1 \leq i \leq m$ to $(L P 1)$. Also, let $(\tilde{u}, \widetilde{v})$ and $\left(\tilde{u}^{\prime}, \widetilde{v}^{\prime}\right)$ be the optimal solutions of the duals of (LP1) and (LP2), respectively, where $(\tilde{u}, \widetilde{v})=\left(\tilde{u}_{1}, \tilde{u}_{2}, \ldots, \tilde{u}_{m}, \widetilde{v}_{1}, \widetilde{v}_{2}, \ldots, \widetilde{v}_{n}\right)$ and $\left(\tilde{u}^{\prime}, \widetilde{v}^{\prime}\right)=\left(\tilde{u}_{1}^{\prime}, \widetilde{u}_{2}^{\prime}, \ldots, \widetilde{u}_{m+1}^{\prime}, \widetilde{v}_{1}^{\prime}, \widetilde{v}_{2}^{\prime}, \ldots, \widetilde{v}_{n+1}^{\prime}\right)$. Then $\tilde{u}_{i}=\widetilde{u}_{i}^{\prime}, 1 \leq i \leq m$ and $\widetilde{v}_{j}=\widetilde{v}_{j}^{\prime}, 1 \leq j \leq n$ provided $\widetilde{u}_{m+1}^{\prime}=\widetilde{0}$.

Proof. The problem (LP2) is:

$$
\begin{aligned}
\text { Minimize } & \sum_{i=1}^{m+1} \sum_{j=1}^{n+1}\left(\widetilde{c}_{i j} \otimes \tilde{x}_{i j}\right), \text { subject to } \\
& \sum_{j=1}^{n+1} \tilde{x}_{i j} \approx \widetilde{a}_{i} \quad(1 \leq i \leq m+1), \\
& \sum_{i=1}^{m+1} \tilde{x}_{i j} \approx \tilde{b}_{j} \quad(1 \leq j \leq n+1), \\
& \tilde{x}_{i j} \succeq 0,
\end{aligned}
$$

where $\tilde{a}_{m+1}=\sum_{i=1}^{m} \tilde{a}_{i}$ and $\tilde{b}_{n+1}=\sum_{i=1}^{m} \tilde{a}_{i} \oplus$ excess supply.

Since $(\underset{\sim}{\tilde{u}}, \widetilde{v})$ is the optimal solution of dual of $(L P l)$, so $\widetilde{u}_{i} \oplus \widetilde{v}_{j} \preceq \widetilde{c}_{i j}, \forall 1 \leq i \leq m, 1 \leq j \leq n$ and $\tilde{u}_{i} \succeq \widetilde{0}, \forall 1 \leq i \leq m$. 
To show that the $\left(\widetilde{u}_{1}^{\prime}, \widetilde{u}_{2}^{\prime}, \ldots, \widetilde{u}_{m}^{\prime}, \widetilde{v}_{1}^{\prime}, \widetilde{v}_{2}^{\prime}, \ldots, \widetilde{v}_{n}^{\prime}\right)$ is also an optimal solution of the dual of $(L P 1)$, it is sufficient to show that

(i) $\tilde{u}_{i}^{\prime} \oplus \widetilde{v}_{j}^{\prime} \preceq \widetilde{c}_{i j}, \forall 1 \leq i \leq m, 1 \leq j \leq n$ and

(ii) $\widetilde{u}_{i}^{\prime} \succeq \widetilde{0}, \forall 1 \leq i \leq m$ and $\widetilde{v}_{j}^{\prime} \succeq \widetilde{0}, \forall 1 \leq j \leq n$.

(i) Since $\left(\widetilde{u}^{\prime}, \widetilde{v}^{\prime}\right)$ is the optimal solution of the dual to problem (LP2), so

$$
\begin{aligned}
& \widetilde{u}_{i}^{\prime} \oplus \widetilde{v}_{j}^{\prime} \preceq \widetilde{c}_{i j}, \forall 1 \leq i \leq m+1,1 \leq j \leq n+1, \\
& \text { so } \widetilde{u}_{i}^{\prime} \oplus \widetilde{v}_{j}^{\prime} \preceq \widetilde{c}_{i j}, \forall 1 \leq i \leq m, 1 \leq j \leq n .
\end{aligned}
$$

(ii) Since $\widetilde{u}_{i}^{\prime} \oplus \widetilde{v}_{j}^{\prime} \preceq \widetilde{c}_{i j}$, we have

$$
\begin{aligned}
& \widetilde{u}_{m+1}^{\prime} \oplus \widetilde{v}_{j}^{\prime} \preceq \widetilde{c}_{(m+1) j} \forall 1 \leq j \leq n+1 \text { and } \widetilde{u}_{i}^{\prime} \oplus \widetilde{v}_{n+1}^{\prime} \preceq \widetilde{c}_{i(n+1)}, \forall 1 \leq i \leq m+1 . \\
& \widetilde{u}_{m+1}^{\prime} \oplus \widetilde{v}_{j}^{\prime} \preceq \widetilde{c}_{(m+1) j} \forall 1 \leq j \leq n+1 \text { implies } \\
& \left.\widetilde{v}_{j}^{\prime} \preceq \widetilde{c}_{(m+1) j} \text { (since } \widetilde{u}_{m+1}^{\prime}=\widetilde{0}\right) \\
& \Rightarrow \widetilde{v}_{j}^{\prime} \preceq \widetilde{c}_{i j} \text { for all } i \text { (since } \widetilde{c}_{(m+1) j}=\min _{1 \leq i \leq m}\left(\widetilde{c}_{i j}\right) \\
& \Rightarrow \widetilde{c}_{i j} \ominus \widetilde{v}_{j}^{\prime} \succeq \widetilde{0} \\
& \left.\Rightarrow \widetilde{u}_{i}^{\prime} \succeq \widetilde{0} \text { (since corresponding to each } \widetilde{v}_{j}^{\prime} \text {, there exist } \widetilde{u}_{i}^{\prime} \text { such that } \widetilde{u}_{i}^{\prime} \oplus \widetilde{v}_{j}^{\prime}=\widetilde{c}_{i j}\right) .
\end{aligned}
$$

Similarly from $\widetilde{u}_{i}^{\prime} \oplus \widetilde{v}_{n+1}^{\prime} \preceq \widetilde{c}_{i(n+1)} \forall 1 \leq i \leq m+1$, it can be proved that $\widetilde{v}_{j}^{\prime} \succeq \widetilde{0}$ (since $\widetilde{u}_{m+1}^{\prime}=\widetilde{0}$, so $\widetilde{v}_{n+1}^{\prime}=\widetilde{0}$ as $\widetilde{b}_{n+1}$ are so chosen that in any feasible solution $\widetilde{x}_{(m+1)(n+1)}$ must be a basic variable).

This completes the proof.

\subsection{Steps of the proposed method}

Step 1: Balance the given FFTP by adding a dummy source $\left((m+1)^{t h}\right.$ source) with availability equal to total availability as well as dummy destination $\left((n+1)^{\text {th }}\right.$ destination) with demand equal to sum of total availability and excess supply. That is

$$
\tilde{a}_{m+1}=\sum_{i=1}^{m} \tilde{a}_{i} \quad \text { and } \quad \tilde{b}_{n+1}=\sum_{i=1}^{m} \tilde{a}_{i} \oplus \text { excess supply. }
$$

The unit transportation costs are taken as follows:

$$
\begin{aligned}
& \widetilde{c}_{i(n+1)}=\min _{1 \leq j \leq n}\left(\widetilde{c}_{i j}\right), 1 \leq i \leq m, \quad \widetilde{c}_{(m+1) j}=\min _{1 \leq i \leq m}\left(\widetilde{c}_{i j}\right), 1 \leq j \leq n, \\
& \widetilde{c}_{i j}=\widetilde{c}_{i j}, 1 \leq i \leq m, 1 \leq j \leq n \quad \text { and } \quad \widetilde{c}_{(m+1)(n+1)}=(0,0,0,0) .
\end{aligned}
$$

Step 2: Apply any of the existing methods (Basirzadeh 2011; Pandian \& Natrajan 2010, 2010a) to the balanced FFTP obtained in Step 1. Let the fuzzy optimal solution obtained be $\tilde{x}_{i j}, 1 \leq i \leq m+1,1 \leq j \leq n+1$.

Step 3: Find the values of all the dual variables $\widetilde{u}_{i}^{\prime}, 1 \leq i \leq m$ and $\widetilde{v}_{j}^{\prime}, 1 \leq j \leq n+1$ by assuming $\widetilde{u}_{m+1}^{\prime}=(0,0,0,0)$ and using the relation $\tilde{u}_{i}^{\prime} \oplus \widetilde{v}_{j}^{\prime}=\widetilde{c}_{i j}^{\prime}$ for basic variables. 
Table 2. Balanced fully fuzzy transportation problem.

\begin{tabular}{lccccc}
\hline $\begin{array}{l}\text { Destinations } \rightarrow \\
\text { Sources } \downarrow\end{array}$ & $D_{1}$ & $D_{2}$ & $D_{3}$ & $D_{4}$ & Fuzzy availability \\
\hline$S_{1}$ & $(4,5,8,15)$ & $(1,1,2,4)$ & $(1,2,3,6)$ & $(1,1,2,4)$ & $(9,11,20,40)$ \\
$S_{2}$ & $(1,1,2,4)$ & $(7,9,12,20)$ & $(4,5,8,15)$ & $(1,1,2,4)$ & $(3,4,7,14)$ \\
$S_{3}$ & $(0,1,1,2)$ & $(7,12,15,26)$ & $(1,1,2,4)$ & $(0,1,1,2)$ & $(3,4,5,8)$ \\
$S_{4}$ & $(0,1,1,2)$ & $(1,1,2,4)$ & $(1,1,2,4)$ & $(0,0,0,0)$ & $(15,19,32,62)$ \\
Fuzzy demand & $(7,12,15,26)$ & $(4,8,10,18)$ & $(2,5,6,11)$ & $(-25,7,39,111)$ & \\
\hline
\end{tabular}

Step 4: By Theorem 1, $\tilde{u}_{i}^{\prime}=\widetilde{u}_{i}, 1 \leq i \leq m$ and $\widetilde{v}_{j}^{\prime}=\widetilde{v}_{j}, 1 \leq j \leq n$. Find those dual variables $\tilde{u}_{i}$ and $\widetilde{v}_{j}$ which have rank zero. Now, the fuzzy optimal solution of the problem in terms of original sources and destinations is obtained as follows:

Let $\tilde{x}_{(m+1) p}$ for some $p$ and $\tilde{x}_{q(n+1)}$ for some $q$ be the basic variables in the fuzzy optimal solution obtained in Step 2. Also, let $\widetilde{u}_{i}$ and $\widetilde{v}_{j}$ have rank zero for $i \in I$ and $j \in J$.

Then increase the value of the basic variable in the cell with $\min _{i \in I} \widetilde{c}_{i p}$ by $\tilde{x}_{(m+1) p}$ and the value of $\min _{j \in J} \widetilde{c}_{q j}$ by $\tilde{x}_{q(n+1)}$ (because availability of only these sources can be increased (Ebrahimnejad $\&$ Nasseri 2009)). Break the tie in minimum value(s) arbitrarily.

If the minimum cost cell is non-basic in the optimal solution obtained in Step 2 then it may become basic in the final solution.

\section{Numerical example}

In this section, the fully fuzzy transportation problem considered in section 4 is solved by the proposed method.

Step 1: Applying Step 1 of the proposed method to the problem considered in section 4, we obtain the balanced FFTP shown in the table 2.

Step 2: Solving this balanced fuzzy transportation problem by using any of the existing methods (Basirzadeh 2011; Pandian \& Natrajan 2010, 2010a), following fuzzy optimal solution is obtained:

$\tilde{x}_{12}=(4,8,10,18), \tilde{x}_{13}=(2,5,6,11), \tilde{x}_{14}=(-102,-25,27,116), \tilde{x}_{21}=(3,4,7,14)$, $\tilde{x}_{31}=(3,4,5,8), \tilde{x}_{41}=(-15,0,7,20), \tilde{x}_{44}=(-5,12,32,77)$.

Table 3. Fuzzy optimal solution for Example 1.

Existing methods (Basirzadeh 2011; Pandian \&

Natrajan 2010, 2010a)

Proposed method

\begin{tabular}{|c|c|}
\hline $\begin{array}{l}\tilde{x}_{11}=(-15,0,7,20), \quad \tilde{x}_{12}=(4,8,10,18) \\
\tilde{x}_{13}=(2,5,6,11), \quad \tilde{x}_{14}=(-40,-12,7,49) \\
\tilde{x}_{21}=(3,4,7,14), \quad \tilde{x}_{31}=(3,4,5,8)\end{array}$ & $\begin{array}{c}\tilde{x}_{12}=(-98,-17,37,134), \quad \tilde{x}_{13}=(2,5,6,11) \\
\widetilde{x}_{21}=(3,4,7,14), \quad \tilde{x}_{31}=(-12,4,12,28)\end{array}$ \\
\hline $\begin{array}{l}\text { Total fuzzy transportation cost } \\
=(-216,26,113,510)\end{array}$ & $\begin{array}{l}\text { Toatl fuzzy transportation cost } \\
\quad=(-411,-6,118,714)\end{array}$ \\
\hline
\end{tabular}


Step 3: Assuming $\widetilde{u}_{4}^{\prime}=(0,0,0,0)$ and using the relation $\tilde{u}_{i}^{\prime} \oplus \tilde{v}_{j}^{\prime}=\widetilde{c}_{i j}$ for the basic variables, the values of dual variables $\tilde{u}_{i}^{\prime},(1 \leq i \leq 3)$ and $\widetilde{v}_{j}^{\prime},(1 \leq j \leq 4)$ and are found to be: $\tilde{u}_{1}^{\prime}=(1,1,2,4), \tilde{u}_{2}^{\prime}=(-1,0,1,4), \widetilde{u}_{3}^{\prime}=(-2,0,0,2), \widetilde{v}_{1}^{\prime}=(0,1,1,2), \widetilde{v}_{2}^{\prime}=(-3,-1,1,3)$, $\widetilde{v}_{3}^{\prime}=(-3,0,2,5), \widetilde{v}_{4}^{\prime}=(0,0,0,0)$.

Step 4: By Step 4 of the proposed method, we find that the $\Re\left(\tilde{u}_{3}\right)=0$ and $\Re\left(\widetilde{v}_{2}\right)=0$ and also there is only single basic variable corresponding to each of $S_{3}$ and $D_{2}$ i.e. $\tilde{x}_{31}$ corresponding to source $S_{3}$ and $\tilde{x}_{12}$ corresponding to destination $D_{2}$. So increase $\tilde{x}_{31}$ and $\tilde{x}_{12}$ by the fuzzy quantities $\tilde{x}_{41}$ and $\tilde{x}_{14}$, respectively. Hence the fuzzy optimal solution is:

$\tilde{x}_{12}=(4,8,10,18) \oplus(-102,-25,27,116)=(-98,-17,37,134), \tilde{x}_{13}=(2,5,6,11), \tilde{x}_{21}$

$=(3,4,7,14), \tilde{x}_{31}=(3,4,5,8) \oplus(-15,0,7,20)=(-12,4,12,28)$.

So, the total fuzzy transportation cost is $(-411,-6,118,714)$.

\section{Conclusions}

The fuzzy optimal solution of Example 1, by the existing methods (Basirzadeh 2011; Pandian \& Natrajan 2010, 2010a) as well as the proposed method is shown in table 3.

From this table, it is clear that the fuzzy optimal solution obtained by the proposed method tells us that the excess availability be supplied to the destinations $D_{1}$ and $D_{2}$. Also, the total transportation cost by the proposed method is less than the existing methods (Basirzadeh 2011; Pandian \& Natrajan 2010, 2010a).

\section{Symbols}

m: number of sources

$\mathrm{n}$ : number of destinations

$\tilde{a}_{i}$ : the fuzzy availability of the product at source $S_{i}$

$\widetilde{b}_{j}$ : the fuzzy demand of the product at destination $D_{j}$

$\widetilde{c}_{i j}$ : the fuzzy cost for transporting one unit of the product from $S_{i}$ to $D_{j}$

$\tilde{x}_{i j}$ : the fuzzy quantity of the product to be transported from $S_{i}$ to $D_{j}$

$\tilde{u}_{i}$ : dual variable corresponding to $i$ th source in $(L P 1)$

$\widetilde{v}_{j}$ : dual variable corresponding to $j$ th destination in $(L P 1)$

$\widetilde{u}_{i}^{\prime}$ : dual variable corresponding to $i$ th source in $(L P 2)$

$\widetilde{v}_{j}^{\prime}$ : dual variable corresponding to $j$ th destination in $(L P 2)$

\section{Acknowledgements}

The authors thank to the Corresponding Editor and the anonymous reviewer for their valuable suggestions which helped in improving the paper. The first author would also like to thank the Council of Scientific and Industrial Research (CSIR), Government of India for providing financial support. 


\section{References}

Appa G M 1973 The transportation problem and its variants. Oper. Res. Quart. 24: 79-99

Arsham H and Khan A B 1989 A simplex-type algorithm for general transportation problems: An alternative to stepping stone. J. Oper. Res. Soc. 40: 581-590

Basirzadeh H 2011 An approach for solving fuzzy transportation problem. Appl. Math. Sci. 5: 1549-1566

Das M K and Baruah H K 2007 Solution of the transportation problem in fuzzified form. J. Fuzzy Math. 15: 79-95

De P K and Yadav B 2010 Approach to defuzzify the trapezoidal fuzzy number in transportation problem. Internat. J. Comput. Cognit. 8: 64-67

Ebrahimnejad A and Nasseri S H 2009 Using complementary slackness property to solve linear programming with fuzzy parameters. Fuzzy Inf. Eng. 3: 233-245

Gani A N, Samuel A E and Anuradha D 2011 Simplex type algorithm for solving fuzzy transportation problem. Tamsui Oxf. J. Math. Sci. 27: 89-98

Hitchcock F L 1941 The distribution of a product from several sources to numerous localities. J. Math. Phys. 20: 224-230

Kaufmann A and Gupta M M 1985 Introduction to Fuzzy Arithmetics: Theory and Applications. Van Nostrand Reinhold New York

Kaur A and Kumar A 2011 A new method for solving fuzzy transportation problems using ranking function. Appl. Math. Model. 35: 5652-5661

Kikuchi S 2000 A method to defuzzify the fuzzy number: Transportation problem application. Fuzzy Sets Syst. 116: 3-9

Liou T S and Wang M J 1992 Ranking fuzzy number with integral value. Fuzzy Sets Syst. 50: 247-255

Pandian P and Natrajan G 2010 A new algorithm for finding a fuzzy optimal solution for fuzzy transportation problems. Appl. Math. Sci. 4: 79-90

Pandian P and Natrajan G 2010a An optimal more-for-less solution to fuzzy transportation problems with mixed constraints. Appl. Math. Sci. 4: 1405-1415

Saad O M and Abbas S A 2003 A parametric study on transportation problem under fuzzy environment. $J$. Fuzzy Math. 11: 115-124

Zadeh L A 1965 Fuzzy sets. Inf. Control, 8: 338-353 